\title{
Drug interactions may be important risk factors for methotrexate neurotoxicity, particularly in pediatric leukemia patients
}

\author{
Victoria J. Forster ${ }^{1} \cdot$ Frederik W. van Delft $^{1} \cdot$ Susan F. Baird $^{2} \cdot$ Shona Mair $^{2}$ • \\ Roderick Skinner ${ }^{3} \cdot$ Christina Halsey $^{4}$
}

Received: 26 May 2016 / Accepted: 2 September 2016 / Published online: 22 September 2016

(C) The Author(s) 2016. This article is published with open access at Springerlink.com

\begin{abstract}
Purpose Methotrexate administration is associated with frequent adverse neurological events during treatment for childhood acute lymphoblastic leukemia. Here, we present evidence to support the role of common drug interactions and low vitamin $\mathrm{B}_{12}$ levels in potentiating methotrexate neurotoxicity.

Methods We review the published evidence and highlight key potential drug interactions as well as present clinical evidence of severe methotrexate neurotoxicity in conjunction with nitrous oxide anesthesia and measurements of vitamin $\mathrm{B}_{12}$ levels among pediatric leukemia patients during therapy.

Results We describe a very plausible mechanism for methotrexate neurotoxicity in pediatric leukemia patients involving reduction in methionine and consequential disruption of myelin production. We provide evidence that a number of commonly prescribed drugs in pediatric leukemia management interact with the same folate biosynthetic pathways and/or reduce functional vitamin $B_{12}$ levels and hence are likely to increase the toxicity of methotrexate in these patients. We also present a brief case study supporting out hypothesis that nitrous oxide contributes to methotrexate neurotoxicity and a nutritional study, showing that
\end{abstract}

Victoria J. Forster

victoria.forster@ncl.ac.uk

1 Paul O'Gorman Building, Northern Institute for Cancer Research, Newcastle University, Framlington Place, Newcastle upon Tyne NE2 4HH, UK

2 Royal Hospital for Sick Children, Edinburgh, UK

3 Great North Children's Hospital, Newcastle upon Tyne, UK

4 Institute of Cancer Sciences, University of Glasgow, Glasgow, UK vitamin $B_{12}$ deficiency is common in pediatric leukemia patients.

Conclusions Use of nitrous oxide in pediatric leukemia patients at the same time as methotrexate use should be avoided especially as many suitable alternative anesthetic agents exist. Clinicians should consider monitoring levels of vitamin $B_{12}$ in patients suspected of having methotrexate-induced neurotoxic effects.

Keywords Neurotoxicity $\cdot$ Hematology $\cdot$ Methotrexate Nitrous oxide $\cdot$ Leukemia $\cdot$ Toxicity

Adverse neurological events are very common during treatment for pediatric acute lymphoblastic leukemia (ALL) and include seizures, stroke-like syndrome and leukoencephalopathy. Recent trials report neurological adverse events in $4-20 \%$ of patients [1, 2]. Additionally, chronic neurotoxicity is emerging as a worrying late effect [3], and 40-60\% of childhood ALL survivors experience neurocognitive difficulties [4], with methotrexate strongly implicated. Despite toxicities, this mainstay of ALL therapy, given intravenously, orally and intrathecally (IT) is credited with driving down the incidence of central nervous system (CNS) relapse without the need for radiotherapy.

Additionally, although rare, neurotoxicity with similar radiological features of leukoencephalopathy has occasionally been reported following oral methotrexate used in patients with autoimmune and inflammatory disorders [5, 6], where neurotoxicity may appear many years into treatment in patients on a stable dose of methotrexate. Genome wide association studies in childhood ALL patients have failed to conclusively identify any predictive genetic markers for methotrexate neurotoxicity [7]. Neurotoxicity is not directly dose related and does not necessarily occur on first 
exposure or on re-exposure after a neurological event [8] as may be expected if attributed to genetic vulnerability. This suggests that additional risk factors may be important.

We propose that methotrexate-induced neurotoxicity may be potentiated by common drug interactions, and/or the presence of low vitamin $\mathrm{B}_{12}$ (cobalamin) levels, which lead to elevated methotrexate levels in cerebrospinal fluid (CSF) or result in synergistic or additive effects on convergent metabolic pathways. A particularly important and under-appreciated drug interaction may be the concomitant use of inhaled nitrous oxide $\left(\mathrm{N}_{2} \mathrm{O}\right)$ and methotrexate, which is common practice in many pediatric hematology centers where lumbar punctures to administer intrathecal (IT) methotrexate are performed under general anesthesia.

A recent case report highlighted the risk of severe neurotoxicity with the combination of $\mathrm{N}_{2} \mathrm{O}$ and methotrexate [9]. Here, we briefly present a second case with multiple acquired risk factors. A 12-year-old girl with acute undifferentiated leukemia received regular IT methotrexate as part of her chemotherapy schedule. All IT therapy was administered under general anesthesia with propofol induction followed by a gaseous mix of $\mathrm{N}_{2} \mathrm{O}$ and oxygen. Other concomitant drugs included the proton-pump inhibitor (PPI) omeprazole. Four days after the 5th dose of IT methotrexate, she was presented with focal seizures rapidly progressing to generalized tonic-clonic seizures, disinhibition, severe agitation and left upper limb (LUL) weakness. MRI (T2/FLAIR and diffusion weighted) imaging on day 2 showed widespread hyperintense subcortical white matter lesions in the frontal and parietal regions with areas of restricted diffusion consistent with leukoencephalopathy. Seizure activity continued for 5 days despite maximal anticonvulsant doses of benzodiazapines and levetiracetam and the radiological changes worsened with increasing cerebral edema and pressure effects requiring dexamethasone. She made a clinical recovery over the next 7 days but with some residual LUL weakness. Serum vitamin $B_{12}$ was measured on recovery (3 weeks from the last IT methotrexate) and was low at $154 \mathrm{ng} / \mathrm{L}$ (normal range $200-1100 \mathrm{ng} / \mathrm{L}$ ).

Methotrexate exerts its anti-leukemic action via inhibition of the enzyme dihydrofolate reductase, ultimately reducing the amount of tetrahydrofolate available for DNA synthesis leading to cell death (summarized in Fig. 1). The reduction in tetrahydrofolate also results in reduced synthesis of methionine from the precursor homocysteine by the enzyme methionine synthase, which requires vitamin $B_{12}$ as a co-factor. Methionine is then converted to S-adenosyl methionine (SAM), a methyl donor which has a critical role in regulating myelin sheath formation and lipid production [10]. This combined with known neuroexcitatory properties of downstream products of homocysteine [11] (Fig. 1) may explain the propensity for CNS side effects in these patients. Indeed, CSF concentrations of SAM were found to be significantly lower in pediatric leukemia patients during methotrexate treatment compared to age-matched controls, whereas levels of myelin basic protein, considered to be a marker of myelin breakdown, were increased [12].

Methionine depletion and accumulation of homocysteine may be potentiated by the co-administration of other medications via two mechanisms; (1) a direct drug interaction leading to increased methotrexate plasma (and/or CSF) concentrations or (2) interference with the same metabolic pathways as methotrexate. Examples of (1) include fluoroquinolone antibiotics, piperacillin (the most commonly prescribed antibiotic for episodes of febrile neutropenia during pediatric leukemia treatment in the UK) and protonpump inhibitors (PPIs). The latter delay plasma elimination of methotrexate leading to renal and liver toxicity [13]. A number of drugs interfere with (2), mainly via depletion of functional vitamin $B_{12}$ (Fig. 1) - as exemplified by nitrous oxide [14]. In addition, PPIs may reduce the bioavailability of dietary vitamin $\mathrm{B}_{12}$ [15] and antimetabolites such as 6-mercaptopurine may cause $\mathrm{B}_{12}$ malabsorption secondary to enteropathy. Indeed, a small pilot study in our local institution recorded low vitamin $\mathrm{B}_{12}$ levels during treatment in 4/19 pediatric patients ( $21 \%$ ) with ALL. Three out of four patients where levels were low experienced severe gastrointestinal enteropathy and 1 patient experienced a severe neurological event. In addition, the same study revealed that $9 / 17$ of the same cohort tested for zinc had clinically low levels at some point during their treatment. Zinc is also a critical co-factor for methionine synthase (Fig. 1); hence, low levels may also contribute to the disruption of this pathway by methotrexate treatment.

The evidence presented above suggests that low $\mathrm{B}_{12}$ levels, recent general anesthesia with nitrous oxide or introduction of additional drugs interacting with the same pathways, may be responsible for the idiosyncratic occurrence of methotrexate neurotoxicity in pediatric leukemia patients undergoing treatment with high doses. We aim to raise awareness globally of this potential interaction and particularly ensure that nitrous oxide anesthesia is avoided in all patients on methotrexate as advised by the British National Formulary [16].

In the era where personalized medicine integrated with genomic approaches is seen as the ultimate goal, it 


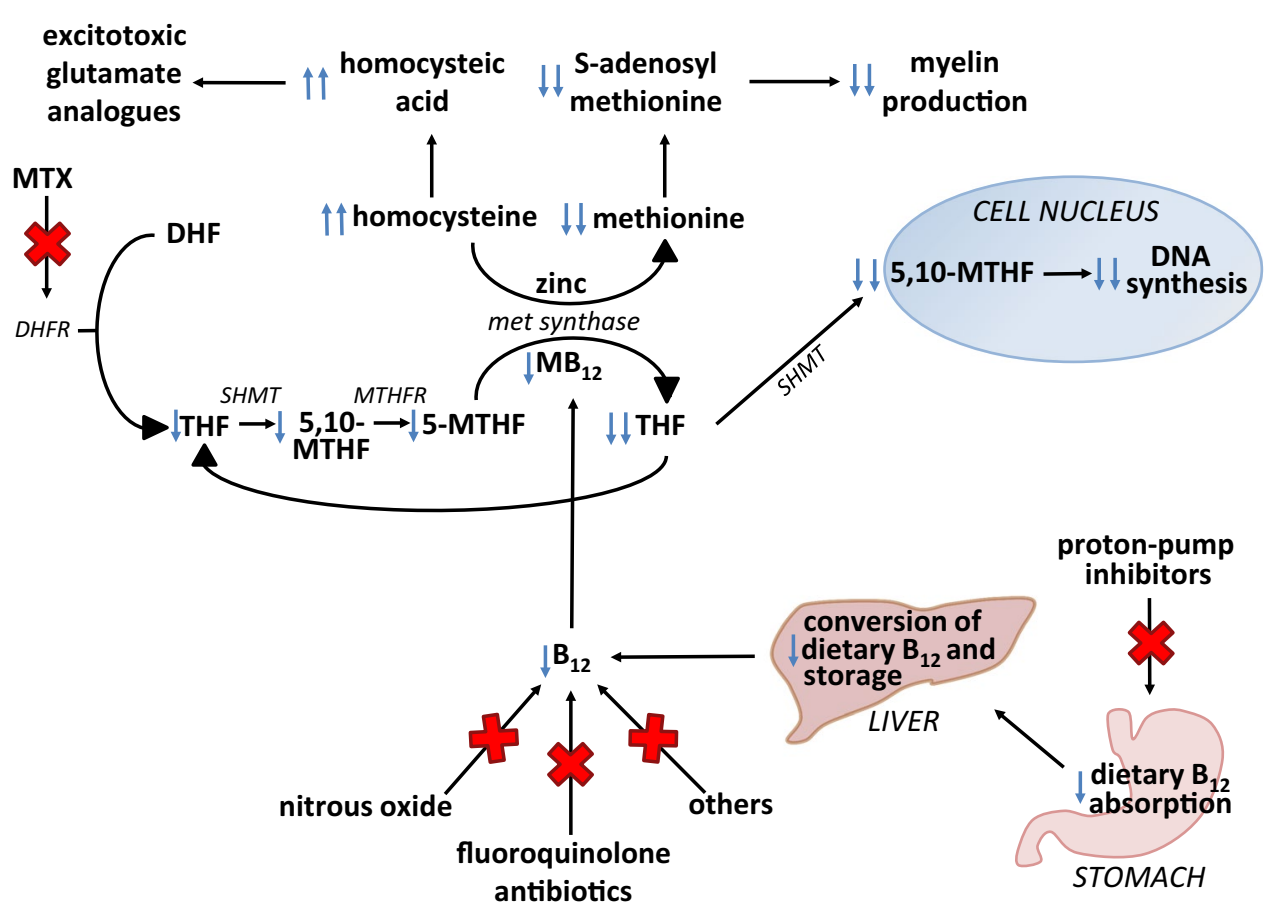

Fig. 1 A summary of the biochemical reactions involving folate and vitamin $B_{12}$ inside an oligodendrocyte and proposed inhibition of myelin production by co-administration of methotrexate (MTX) and drugs affecting vitamin $\mathrm{B}_{12}$. Abbreviations: 5-MTHF (5-methyltetrahydrofolate, levomefolic acid), $M B_{12}$ (methyl $\mathrm{B}_{12}$ ), THF (tetrahydrofolate, tetrahydrofolic acid), 5,10-MTHF (5,10-methylene THF), $D H F$ (dihydrofolate, dihydrofolic acid), DHFR (dihydrofolate reductase), $M B_{12}$ (methyl-vitamin $\mathrm{B}_{12}$ ), MTHFR (methylenetetrafolate reductase), MTX (methotrexate), met synthase (methionine synthase), SHMT (serine hydroxyl-methyltransferase). MTHF participates in the production of methionine from homocysteine by methionine synthase, catalyzed by $\mathrm{MB}_{12}$ and zinc, creating THF and methionine. THF participates in the production of purines and pyrimidines for DNA synthesis. Methionine is a vital amino acid involved in myelin production via its conversion to S-adenosyl methionine (SAM). SAM is involved in the methylation of many proteins and intermediates ultimately involved in myelin production, such as phosphati-

is important not to overlook 'old-fashioned' drug interactions, especially if such risks can easily be minimized by a change in drug scheduling or use of suitable alternative agents.

Acknowledgments We thank the contributing patient and family for allowing consent for her clinical information to be presented within this manuscript. We thank the relevant clinical team for sharing this information and also Raquel Renevuelta-Iniesta for sharing the preliminary results on vitamin $\mathrm{B}_{12}$ levels in pediatric patients undergoing treatment for ALL. VJF is also grateful to Dr Andrew McKendry and the late Dr Judith Kingston for early support with idea generation and research design.

Author contributions VJF conceived of the original idea and wrote the paper. $\mathrm{CH}$ wrote the paper. SFB contributed the clinical case study information. SM contributed the vitamin $\mathrm{B}_{12}$ nutritional study dylcholine, which is important in the production of sphingomyelin, a major component of the myelin sheath. Homocysteine can be converted to homocysteic acid and homocysteine sulfinic acid which are excitotoxic glutamate analogues acting at the N-methyl-D-aspartate (NMDA) receptor, which may be a factor in acute methotrexateinduced neurotoxicity. Methotrexate inhibits the function of DHFR, preventing the conversion of DHF to MTHF. Active vitamin $\mathrm{B}_{12}$ contains reduced cobalt $\left(\mathrm{Co}^{+}\right)$, but nitrous oxide $\left(\mathrm{N}_{2} \mathrm{O}\right)$ produces irreversible oxidation to $\mathrm{Co}^{++}$and $\mathrm{Co}^{+++}$, rendering vitamin $\mathrm{B}_{12}$ inactive. Any simultaneous compromise of folate and vitamin $B_{12}$ via co-administration of methotrexate and agents known to deplete active vitamin $\mathrm{B}_{12}$, such as $\mathrm{N}_{2} \mathrm{O}$ could result in increased homocysteine and reduced methionine levels both of which may contribute to the neurotoxic effects of methotrexate treatment. Other as yet unidentified compounds may also reduce bioavailable vitamin $\mathrm{B}_{12}$ levels. Blue arrows indicate proposed increase or reduction in various relevant pathway metabolites

information. All authors contributed ideas and expertise and contributed to the final version of the manuscript.

Funding This work was supported by the JGW Patterson Foundation, Newcastle upon Tyne, UK, (VF) and Children with Cancer UK $(\mathrm{CH})$.

\section{Compliance with ethical standards}

Conflict of interest All authors declare that they have no conflicts of interest.

Open Access This article is distributed under the terms of the Creative Commons Attribution 4.0 International License (http://creativecommons.org/licenses/by/4.0/), which permits unrestricted use, distribution, and reproduction in any medium, provided you give appropriate credit to the original author(s) and the source, provide a link to the Creative Commons license, and indicate if changes were made. 


\section{References}

1. Vora A, Goulden N, Wade R et al (2013) Treatment reduction for children and young adults with low-risk acute lymphoblastic leukaemia defined by minimal residual disease (UKALL 2003): a randomised controlled trial. Lancet Oncol 14(3):199-209

2. Bhojwani D, Sabin ND, Pei D et al (2014) Methotrexate-induced neurotoxicity and leukoencephalopathy in childhood acute lymphoblastic leukemia. J Clin Oncol 32(9):949-959

3. Schuitema I, Deprez S, Van Hecke W et al (2013) Accelerated aging, decreased white matter integrity, and associated neuropsychological dysfunction 25 years after pediatric lymphoid malignancies. J Clin Oncol 31(27):3378-3388

4. Van der Plas E, Nieman BJ, Butcher DT, Hitzler JK, Weksberg R, Ito S, Schachar R (2015) Neurocognitive late effects of chemotheapy in survivors of acute lymphoblastic leukemia: focus on methotrexate. J Can Acad Child Adolesc Psychiatry 24(1):25-32 (Winter)

5. Raghavendra S, Nair MD, Chemmanam T, Krishnamoorthy T, Radhakrishnan VV, Kuruvilla A (2007) Disseminated necrotizing leukoencephalopathy following low-dose oral methotrexate. Eur J Neurol 14(3):309-314

6. Gonzazlez-Suarez I, Aquilar-Amat MJ, Triqueros M, Borobia AM, Cruz A, Arpa J (2014) Leukoencephalopathy due to oral methotrexate. Cerebellum 13(1):178-183

7. Radtke S, Zolk O, Renner B et al (2013) Germline genetic variations in methotrexate candidate genes are associated with pharmacokinetics, toxicity and outcome in childhood acute lymphoblastic leukemia. Blood 121(26):5145-5153

8. Badke C, Fleming A, Iqbal A, Khilji O, Parhas S, Weinstein J, Morgan E, Hiiya N (2015) Rechallenging with intrathecal methotrexate after developing subacute neurotoxicity in children with hematologic malignancies. Pediatr Blood Cancer 63(4):723-726

9. Lobel U, Trah J, Escherich G (2015) Severe neurotoxicity following intrathecal methotrexate with nitrous oxide sedation in a child with acute lymphoblastic leukemia. Pediatr Blood Cancer 62(3):539-541

10. Chamberlin ME, Ubagai T, Mudd SH, Wilson WG, Leonard JV, Chou JY (1996) Demyelination of the brain is associated with methionine adenosyltransferase I/III deficiency. J Clin Invest 98(4):1021-1027

11. Vijayanathan V, Gulinello M, Ali N, Cole PD (2011) Persistent cognitive deficits, induced by intrathecal methotrexate, are associated with elevated CSF concentrations of excitotoxic glutamate analogs and can be reversed by an NMDA antagonist. Behav Brain Res 225(2):491-497

12. Surtees R, Clelland J, Hann I (1998) Demyelination and single-carbon transfer pathway metabolites during the treatment of acute lymphoblastic leukemia: CSF studies. J Clin Oncol 16(4):1505-1511

13. Suzuki K, Doki K, Homma M et al (2009) Co-administration of proton pump inhibitors delays elimination of plasma methotrexate in high-dose methotrexate therapy. Br J Clin Pharmacol 67(1):44-49

14. Deacon R, Lumb M, Perry J et al (1978) Selective inactivation of vitamin B12 in rats by nitrous oxide. Lancet 2(8098):1023-1024

15. McColl KE (2009) Effect of proton pump inhibitors on vitamins and iron. Am J Gastroenterol 104(Suppl 2):S5-S9

16. Joint Formulary Committee. British National Formulary (online) London: BMJ Group and Pharmaceutical Press. http://www. medicinescomplete.com. Accessed 5 Feb 2016 